\title{
Curieux, curieusement, curiosité
}

\section{Citation}

Blair, Ann. 2003. Curieux, curieusement, curiosité. In Le Dictionnaire universel de Furetière, ed. Hélène Merlin, a special issue of Littératures classiques 47: $101-7$.

\section{Permanent link}

http://nrs.harvard.edu/urn-3:HUL.InstRepos:29669480

\section{Terms of Use}

This article was downloaded from Harvard University's DASH repository, and is made available under the terms and conditions applicable to Open Access Policy Articles, as set forth at http:// nrs.harvard.edu/urn-3:HUL.InstRepos:dash.current.terms-of-use\#OAP

\section{Share Your Story}

The Harvard community has made this article openly available.

Please share how this access benefits you. Submit a story.

Accessibility 
manuscript for:

Ann Blair, "Curieux, curieusement, curiosité," in Le Dictionnaire universel de Furetière, ed. Hélène Merlin, a special issue of Littératures classiques 47 (2003), pp. 101-7.

Ann Blair

\section{Curieux, curieusement, curiosité}

Le Furetière présente un triple intérêt pour l'étude du thème de la curiosité. Dans la page de définitions consacrée à ces termes il rend compte d'une pluralité de sens et de connotations qui témoigne d'une évolution linguistique et conceptuelle en cours à la fin du 17 e siècle. De plus, Furetière accumule plus de 200 références à ces termes dans les exemples qu'il donne pour expliquer d'autres mots; cette récurrence remarquable (le Dictionnaire de l'Académie par contraste n'emploie ces termes qu'une trentaine de fois au total) donne une riche perspective sur les curieux et leurs habitudes tels que les perçoit Antoine Furetière'. Enfin, Furetière lui-même s'adonne à la curiosité, en utilisant le terme ou non, lorsqu'il présente dans certains articles, notamment en sciences naturelles, des informations détaillées qui vont au-delà des besoins stricts du dictionnaire.

Une excellente étude récente nous permet de placer les définitions que donne Furetière de "curieusement, curieux, curiosité" dans le contexte d'une plus longue histoire de l'évolution de cette famille de motsii. Formés du latin "curiosus" et "curiositas", "curieux" et "curiosité" restent sans doute des latinismes d'emploi plutôt rare en français avant l'époque moderne. Leur sens originel, du latin "cura"--soin, attention--est encore présent, surtout en français, notamment dans le sens adverbial, mais aussi comme dernier sens pour l'adjectif: 
Curieusement. D'une maniere curieuse, exacte....

Curieux, signifie aussi, Propre, bien net, bien vestu. ...

Parmi les exemples Furetière a dû surtout aimer son emploi paradoxal: "on dit ironiquement d'un homme malpropre, qu'il est curieux en linge sale" puisqu'on retrouve cet exemple encore sous "linge" et sous "sale"

Les autres définitions de cette famille de termes tournent autour du sens qui nous est plus familier: "curieux, adj. et subst. Celuy qui veut tout sçavoir, et tout apprendre. Tous les hommes ont un desir curieux de sçavoir l'avenir." Furetière note immédiatement les diverses connotations possibles: "Il se prend quelquefois en mauvaise part. C'est un indiscret qui est curieux de sçavoir les secrets d'autruy, qui decachette les lettres. ... Se dit en bonne part de celuy qui a desir d'apprendre, de voir les bonnes choses, les merveilles de l'art et de la nature." La différence tient surtout au type de savoir recherché: "Il ne faut point etre curieux d'apprendre les affaires des Princes quand ils les cachent. Cervantes a écrit l'Histoire du Curieux impertinent, qui vouloit esprouver si sa femme luy étoit fidelle." La curiosité légitime doit reconnaître selon ces définitions une sphère privée non seulement pour les grands, mais aussi pour les petits, et même les femmes. On trouve dans l'édition de 1701 des citations de moralistes, à dominante négative, ajoutées aux articles "curieux" et "curiosité". iv Le jeu entre connotations favorables et défavorables était donc flexible et pouvait être équilibré diversement; les modifications apportées par Basnage de Beauval en 1701 renchérissent la conclusion que Furetière pour sa part a mis l'accent sur les aspects positifs de la curiosité.

Furetière se trouve au milieu d'une évolution linguistique et culturelle complexe qui transforma la curiosité de vice traditionnel en vertu possible, voire probable. La connotation de la curiosité était exclusivement négative au moyen age, suivant les jugements sévères des Pères de l'Eglise 
sur la vanité de tout savoir non religieux. Pour l'encyclopédiste Gregor Reisch, par exemple, dans sa somme du savoir médiéval en 1503 , le vice de la curiosité était opposé à la vertu de la $\underline{\text { studiositas, }}$ qui dirigeait toute scientia vers la connaissance de Dieuvv. La curiosité acquiert rapidement des connotations plus favorables entre Reisch et Furetière, parallèlement au développement des pratiques de la collection de curiosités par les grands et les moins grands ${ }^{\mathrm{vi}}$. Mais les connotations négatives initiales perdurent. On les reconnaît en Angleterre au 17e siècle lorsqu'elles motivent la rhétorique de la théologie naturelle qui justifie comme pieuses des recherches scientifiques qui pourraient sembler vaines, au 18e lorsqu'on appelle curieux surtout ceux qui sont perçus comme des arrivistes sociaux et encore aujourd'hui dans plusieurs expressions courantes ${ }^{\text {vii. }}$.

Furetière consacre beaucoup de place aux connotations positives de la curiosité: désir de savoir qui incite au voyage, à l'étude de la nature, de l'art, des livres, à la collection des médailles, des estampes, des antiquités, des tableaux. La curiosité louable est tournée sur un sujet légitime, surtout scientifique ou naturel; pourtant cette légitimation est moins souvent justifiée par l'utilité du sujet que par sa "beauté". Ainsi Furetière met l'accent sur les belles choses étudiées: "un curieux qui a feuilleté tous les bons Livres, tous les Livres rares. C'est un Chymiste curieux qui a fait de belles experiences, de belles descouvertes." Parmi les bonnes "sciences curieuses" que Furetière distingue des "vaines" (astrologie, géomance ou magie) il cite encore "la Chymie, une partie de l'Optique, qui fait voir des choses extraordinaires avec des miroirs et des lunettes." Les références à la curiosité mais surtout aux curieux dans plus de deux cents exemples cités dans d'autres définitions révèlent la dominance de cette acceptation récente du curieux qui est valorisé pour la qualité de ce qu'il collectionne et connaît, mais que l'on moque s'il n'a que de piètres pièces. Pour mériter l'admiration le curieux doit avoir collectionné de véritables "curiosités". 
Furetière constate pour finir ce sens de "curiosité" d'origine récente: la curiosité est passée d'un désir subjectif à un sens objectif: "se dit aussi de la chose même qui est rare, secrette, curieuse."

Parmi les nombreuses occurrences de cette famille de termes dans les exemples introduits dans d'autres définitions, il faut mentionner d'abord quelques exemples de "curieux" au sens fort de "soucieux": "les Grecs estoient plus curieux observateurs des accens que les François" ("accent"), les Indiens ramassent curieusement les fleurs de l'"arbre triste", les "antidotes" et "electuaires" sont composés de simples "curieusement choisis". Dans ce même sens d'attentif, on trouve la plupart des références à la curiosité chez les femmes: l'exemple de Popea la femme de Néron, "qui fut plus curieuse de se parer que toutes les autres femems" ("masque", "poupée"); les femmes en général sont dites curieuses d'orner leurs maisons de riches meubles etc ("orner"). L'autre occurrence de la curiosité féminine, attendue elle aussi, est l'exemple de la femme curieuse de nouvelles, dite la gazette du quartier ("à quartier"); de même le peuple est curieux de nouvelles ("nouvelles"). Mais on remarque l'absence de condamnation explicite de ces conduites. La connotation négative de la curiosité indiscrète se retouve dans "enquérant", terme qui "se prend d'ordinaire en mauvaise part", et plus implicitement dans "épiloguer" ("censurer, rechercher curieusement ce qu'il y a de mal dans les actions d'autruy") ou controlleur ("se dit aussi des curieux et critiques, qui observent les actions d'autruy pour les blasmer et les reprendre"). "Furet", "fureter", "fureteur" mettent aussi à jour des choses secrètes, mais l'exemple est favorable: "à force de fureter, ce Marchand a fait un cabinet fort curieux et à bon marché." La seule critique explicite de la curiosité mal placée se trouve sous "obscurcir" où Furetière donne comme exemple: "Les curieux et les mesdisans taschent d'obscurcir la gloire des grands hommes." 
Si Furetière n'insiste pas sur la condamnation traditionnelle de la curiosité indiscrète, les descriptions négatives des curieux chez Furetière tournent pour la plupart autour de leur incapacité à trouver des objets de mérite. Ainsi les exemples abondent de curieux qui n'ont rien que "babioles", "brimborions", "breluques", "colifichets", "godenots", "guenilles", "gueuseries", "racaille", qui n'ont que des tableaux "communs" ou qui ne lisent que des livres frippés ("bouqiner"). Pourtant Furetière défend les curieux contre l'appellation "odieuse" de "marmotiers": "marmot" est "le nom injurieux que donne le peuple ... faute de s'y connaître" aux bustes de bronze et de marbre que collectionnent certains curieux.

La part prépondérante des références aux curieux est favorable. Certes la curiosité est une passion que l'on cherche à "satisfaire" ("naître", "donner dans", "satisfaire," "voilà"). On peut être "fou" ou "friand" de curiosités, objets de collection dans un cabinet; Furetière accumule les exemples de passion pour les tableaux en particulier, qui excitent la "fureur" des curieux, devant lesquels ils "s'escrient" ou "s'extasient", dont certains sont même "idolâtres." Quelquefois Furetière trouve ces passions étranges, comme la manie des tulippes ou pour les pigeons couleur soupe de lait ("tulippe", "soupe"). Mais en général la curiosité suscite des conduites louables. Les curieux constituent des cabinets et développent une expertise particulière.

Fidèle à sa mission d'élucider les termes des arts et métiers, Furetière signale plusieurs termes spécialisés qu'emploient les curieux. D'abord les curieux maitrisent la disposition du cabinet ("accommodement") et emploient pour ce faire des meubles particuliers, dont des "layettes" (tiroirs) et des "tablettes". Ensuite ils ont des termes d'art pour désigner certains objets précieux: "pagode" pour les "petites idoles de porcelaine qui viennent de la Chine," "porcelaine" pour "des coquilles tachetées" et Furetière donne une longue liste de noms de "poires" et de "prunes" que l'on trouve dans les jardins des curieux. Enfin, pour se désigner eux-mêmes, ils empruntent de 
l'Italien le terme de "vertueux" qui "se dit parmi les curieux de ceux qui s'adonnent à la recherche des belles choses, qui ont du goust pour les arts, les sciences et les curiositez naturelles." Le terme fut plus répandu en Angleterre sous sa forme originale ${ }^{\text {viii }}$.

L'emploi de termes d'art par les curieux est le résultat de connaissances et de pratiques spécialisées que Furetière mentionne aussi: les curieux des tulippes appréhendent bien les "mulots", les curieux des tableaux reconnaissent la "manière" des peintres, les curieux fleuristes s'y connaissent en "papillons" et en "vers". Furetière donne même l'exemple d'un curieux qui est "un sçavant Antiquaire qui déchiffre toutes les plus vieilles inscriptions, les titres les plus corrompus et les plus effacez" ("déchiffrer"); plusieurs ont écrit sur les blasons "en curieux et en historiens" ("blasons"). Mais en général une distinction est maintenue entre le curieux qui est un "repertoire de medailles, d'antiquitez" et le savant qui est "un repertoire d'histoires, d'observations, de critiques" ("repertoire"). Ainsi, le cabinet du curieux est un lieu de sociabilité où "les curieux gardent, vendent et troquent toutes sortes de curiosités" alors que le cabinet du savant est un lieu d'étude solitaire: "ce Sçavant est toujours enfermé dans son cabinet" ("cabinet").

Le curieux n'est donc pas souvent savant, mais peut être "un fort bon connoisseur" ("connoisseur"). Pourtant cette connaissance est vulnérable à la passion: "les curieux se trompent souvent en tableaux, et prennent des copies pour des originaux" ("original"). Son activité d'achat et d'échange apparente aussi le curieux au brocanteur (voir "pasté" et "pendant", objets qui se vendent ensemble). Les curieux et les frippiers courent les "inventaires" ou ventes publiques. Furetière observe de plus que les curieux négocient partie en argent, partie en troc ("partie", "troc").

L'attrait du curieux repose avant tout dans sa collection où l'on peut voir toutes sortes de choses 
intéressantes, pour employer le terme d'aujourd'hui, qui remplaça "curieux" à partir du 18e siècle ${ }^{\mathrm{ix}}$. Furetière note dans le cas de plusieurs choses rares que l'on peut en voir des exemples chez les curieux: notamment des pierres abracax ("abracadabra"), des "fetus" conservés dans de l'eau de vie, des "jeux de la nature" et des "singularitez" en général, des "lacrymatoires" (où l'on collectionnait les larmes versées aux funérailles de quelqu'un), des "lampes" antiques, des tasses supposées être tombées de l'"arc-en-ciel", des "petrifications", des mains de "sirene", des "squelettes" et bien sûr des cornes de "licorne", même si Furetière note la conclusion de La Peyrere que ces "cornes" sont en fait des dents de narwhal. Le cabinet du curieux est le dépositaire d'une myriade de specimens rares que l'on ne peut voir que là.

La collection du curieux est souvent estimée pour la beauté des objets qu'on y trouve dans les exemples de Furetière. Ainsi les estampes sont "une belle curiosité", ainsi que les coquilles par leur diversité merveilleuse et les coquillages exquis qu'ont les curieux ("estampes", "coquille", "coquillage") $)^{\mathrm{x}}$. Mais les tableaux sont "la plus belle curiosité"; les curieux embellissent leurs collections de tableaux, qu'ils "ont grand soin de bien embordurer" ("tableau", "embellir", "embordurer"). Les curieux ont aussi de beaux dessins au crayon, des estampes de deux couleurs, des dessins de grands peintres ("crayon", "clair-obscur", "dessein"); ils déterrent les "beaux monuments avec soin" ("déterrer").

Associée à la beauté et à la rareté, la curiosité reste la plupart du temps opposée à l'utilité. Ainsi "l'Algebre est une science curieuse mais elle n'apporte aucun profit ni utilité" ("utilité"); "la pluspart des propositions en Geometrie ne sont d'aucun usage, ne sont qu'une simple curiosité" ("usage"); "on appelle proverbialement des fantaisies musquées, en general toutes sortes de petits bigeous inutiles qui ne servent qu'à la propreté ou à la curiosité" ("musqué"); l'argent mignon est celui qu'on dépense pour "des curiositez ou des vanitez qui ne sont point necessaires à la vie" 
("mignon"). De même on garde les animaux à la ménagerie ou les oiseaux à la volerie, on observe les travaux d'un siège ou on voyage par curiosité ("ménagerie", "volerie," "travail", "voyageur"), c'est-à-dire sans but pratique. La curiosité désigne certes parfois des recherches scientifiques dirigées, telle l'études des "graines" par Mr Grew, ou des "couvées" cassées par Malpighi, des observations de "puces" ou de "moisissure" sous le microscope par Mr Hook. Celles-ci ne constituent jamais cependant une recherche de l'utile. Ce n'est sans doute pas une critique de la curiosité de la dissocier du travail à but lucratif, des activités mercantiles et mécaniques, et de l'associer au contraire à l'otium de la personne qui a du temps, de l'argent et de la passion à diriger au-delà des nécessités de la vie.

Furetière lui-même est conscient de s'être consacré à plusieurs lectures et observations curieuses dans son travail de lexicographe et il sait diriger le lecteur intéressé à ses sources. Ainsi il mentionne fréquemment des livres, des dissertations, des traités curieux (voir, entre beaucoup d'autres exemples, "foudre", "hemine", "insecte" "loxodromies", "sabbathine"). Après un long paragraphe sur la lèpre il explique que "ces remarques curieuses sur lesquelles on s'est un peu estendu à cause de la rareté de la maladie" proviennent de plusieurs auteurs anciens et modernes, de Galien à Anbroise Paré ("lèpre"). Ou bien Furetière tire plusieurs détails du livre sur la mortalité de Jean Graunt qu'il dit contenir "quantité d'autres recherches curieuses" ("mortalité"). Du castor disséqué à l'Académie des Sciences à la giraffe que la plupart des curieux considèrent comme chimérique ("castor", "giraffe"), Furetière porte une attention particulière aux sujets d'histoire naturelle. Au-delà des définitions et des observations sur les mots, leurs origines et leur emploi dans des tournures proverbiales, que l'on pourrait d'ailleurs souvent qualifier de "curieuses", le Dictionaire accumule une grande diversité de connaissances sur les choses. Pierre 
Bayle vante précisément cette qualité encyclopédique du Furetière: "on ramasse cent belles curiositez de l'histoire naturelle, de la physique experimentale et de la pratique des arts. Ce ne sont pas de simples mots qu'on nous enseigne, mais une infinité de choses, mais les principes, les règles et les fondemens des arts et des sciences" (sig. *3v).

Le Furetière constitue ainsi à plusieurs égards l'équivalent livresque d'un cabinet de curiosité. L'ordre alphabétique qu'il suit est tout aussi arbitraire que la disposition typique des cabinets, peu systématique et donnant lieu à des juxtapositions inattendues. Dans le Dictionaire les exemples et les amplifications sont dispersés de façon imprévisible, si bien que l'on trouve "dans chaque page beaucoup de diversite" et on ne peut y faire "beaucoup de chemin sans aprendre quelque chose qui en vaut la peine" (sig. *3v). Le livre permet de rassembler à moindre frais et en moins de place qu'un véritable cabinet une gamme surprenante de descriptions et de rapports de faits que le lecteur est invité à apprécier au gré de lectures ponctuelles ou suivies. C'est un cabinet que l'on peut voir et revoir à sa guise, où l'on retrouve une grande diversité de choses "belles" rapportées sans but utilitaire immédiat--c'est-à-dire des curiosités. Le livre qui imite et rend compte des cabinets de curiosités promet de plaire non seulement à ceux qui connaissent ces lieux euxmêmes, mais aussi au public bien plus large de ceux qui auraient voulu y avoir accès. Il n'est pas étonnant que Furetière, qui pratique ainsi la curiosité au fil de la composition de son ouvrage, mette l'accent sur la connotation positive de cette famille de termes, bien qu'il décrive de façon équilibrée de leurs emplois négatifs. Pour Furetière "curieusement", "curieux" et "curiosité" sont identifiés surtout avec des cabinets remplis de choses rares et belles et des observations qui méritent l'attention de ses lecteurs. L'ouverture de Furetière lui-même à l'exercice de la curiosité, en poursuivant dans son dictionnaire quantité de questions au-delà de ce qui était nécessaire à une définition linguistique même de termes techniques, fait de son 
ouvrage une source particulièrement riche pour l'historien. Il nous permet notamment, entre autres fins, de mieux cerner les connotations et les pratiques de la curiosité vers 1690.

Ann Blair, Dept of History, Harvard University

i Ma recherche sur l'emploi de ces termes en dehors de leurs définitions explicites repose non seulement sur les rapports de mes co-contributeurs à ce volume, que je tiens à remercier de leur travail, mais aussi sur l'emploi du CD-ROM L'atelier historique de la langue française. L'étude du texte ancien reste cependant essentielle; toutes mes citations proviennent du Dictionaire universel (La Haye et Rotterdam: Arnout et Reinier Leers, 1690), 3 vols. ii Voir Neil Kenny, Curiosity in Early Modern Europe. Word Histories (Wiesbaden: Harrassowitz, 1998), surtout pp. 40-41.

iii Pour alléger la discussion je signalerai souvent entre guillemets l'entrée du Dictionaire dont il est question.

iv Voir le Dictionnaire universel recueilli par feu Messire Antoine Furetiere, seconde edition, revue, corrigée et augmentée par Monsieur Basnage de Beauval (La Haye et Rotterdam: Arnoud et Reinier Leers, 1701).

v Gregor Reisch, Margarita philosophica (Freiburg, 1503), livre XII, ch. 46, pp. 538-39. Pour une étude utile des condamnations anciennes de la curiosité, non seulement patristiques, mais aussi païennes, voir André Labhardt, "Curiositas: notes sur l'histoire d'un mot et d'une notion," Museum helveticum 17 (1960), pp. 206-24.

vi Pour des études du phénomène social de la curiosité voir notamment Krzysztof Pomian, Collectionneurs, amateurs et curieux: Paris, Venise XVIe-XVIIIe siècles (Paris: Gallimard, 1987); Antoine Schnapper, Collections et collectionneurs dans la France du XVIIe siècle, 2 vols. (Paris: Flammarion, 1988 et 1994); Katie Whitaker, "The Culture of Curiosity," in Cultures of Natural History, ed. N. Jardine, et al. (Cambridge: Cambridge University Press, 1996), pp. 75-90. Sur les attitudes intellectuelles voir La curiosité à la Renaissance, ed. Jean Céard (Paris: Société d'édition de l'enseignement supérieur, 1986); Curiosité et libido sciendi de la Renaissance aux Lumières, ed. Nicole Jacques-Chaquin et Sophie Houdard (Fontenay-aux-Roses: ENS editions, 1998); Peter Harrison, "Curiosity, Forbidden Knowledge and the Reformation of Natural Philosophy in Early Modern England," Isis 92 (2001): 265-90.

vii Barbara Benedict, Curiosity: a cultural history of early modern inquiry (Chicago: University of Chicago Press, 2001).

viii Sur ce terme voir Walter E. Houghton, Jr., "The English Virtuoso in the Seventeenth Century," Journal of the History of Ideas 3 (1942), pp. 51-73, 190-219.

ix Kenny, pp. 141 et suivantes.

x $\quad$ "Exquis" devient même synonyme de "curieux" ("exquis"). 